\title{
Implicit local refinement for evanescent layers combined with classical FDTD
}

\author{
Wouter Tierens, Daniël De Zutter, Fellow, IEEE,
}

\begin{abstract}
In this paper we hybridize the well-known FDTD method with the fully implicit method of [1]. In effect, this enables local space refinement without necessitating a smaller time step. In particular, this is very useful for thin layers of highly conducting material or to treat complex media allowing evanescent waves such as plasma.
\end{abstract}

Index Terms-FDTD spatial refinement, Budden tunneling, FDTD thin conducting layers

\section{INTRODUCTION}

$\mathbf{I}_{\mathrm{r}}$ $\mathrm{N}$ FDTD, modeling local features where the wave amplitude changes very quickly, on scales far smaller than the overall discretisation length $\Delta_{F D T D}$ is problematic. Such features can be caused by the presence of an actual layer of conducting material with very small skin depth, or by specific properties of the dispersion relation in a non-trivial material such as a plasma.

Most solutions to this problem exploit specific knowledge of the problem at hand and start from a known analytical solution [2], [3]. If no analytical solution is known, local refinement [4] seems like an attractive option were it not that this usually requires a significantly reduced time step (though several "multirate" refinement techniques have been proposed [5], [6] and appendix and several references in [4]).

In this work, we develop a hybrid implicit/explicit method which enables local refinement without requiring a smaller time step. The explicit (FDTD) part can be run at the usual FDTD Courant limit. The implicit part is unconditionally stable and can be run at the very same time step (despite possibly having a far smaller space step). The set of equations which needs to be solved for the implicit part is only as large as the implicit part itself. In this short contribution, only the one-dimensional case is treated allowing to clearly explain the method. Further work aims at extending the approach to higher dimensions.

We apply our new method to solve the well-known problem of transmission at a thin layer of conducting material, both with constant and position-dependent conductivity, thereby demonstrating that this method could just as easily be used to solve problems involving non-homogeneous materials for which no analytical solution is known. As a final example, we solve the "Budden tunneling equation", a wave equation with strongly place-dependent material properties, which is useful in plasma physics but still allows an analytical solution, allowing to further verify the correctness and to demonstrate the versatility of the proposed method.

We start by providing a short introduction to the FDTD method and the fully implicit method in section II. The new hybrid method is presented in section III, followed by some numerical examples in section IV. Conclusions are formulated in section $\mathrm{V}$.

\section{A SHORT PRIMER ON EXPLICIT AND IMPLICIT METHODS}

The FDTD method [7], [4] is extremely well-known and needs no introduction. It is a discretisation of Maxwell's equations on staggered "Yee" cells, such that every derivative (spatial and temporal) has a natural second-order accurate finitedifference analogon precisely where needed (left of figure 1). In figure 1 the arrows represent the $E_{z}$ electric fields and the dots the $B_{y}$ magnetic inductions of our one-dimensional model with propagation along $x$. In FDTD, Faraday's law is indeed enforced by obtaining the E-field spatial derivative by subtracting two spatially separated but simultaneous $E_{z}$-values and the B-field time-derivative by subtracting two temporarily separated but co-located $B_{y}$-values:

$$
\begin{gathered}
\frac{1}{\Delta_{t}}\left(B_{y}\left(x, t+\Delta_{t}\right)-B_{y}(x, t)\right)= \\
\frac{1}{\Delta}\left(E_{z}\left(x+\Delta / 2, t+\Delta_{t} / 2\right)-E_{z}\left(x-\Delta / 2, t+\Delta_{t} / 2\right)\right)
\end{gathered}
$$

The fully implicit method of [1], on the other hand, handles every derivative implicitly (right of figure 1) using four field values of $E_{z}$ and four field values of $B_{z}$ :

$$
\begin{aligned}
\frac{1}{\Delta_{t}}( & \frac{1}{2}\left(B_{y}\left(x, t+\Delta_{t}\right)+B_{y}\left(x+\Delta, t+\Delta_{t}\right)\right) \\
& \left.-\frac{1}{2}\left(B_{y}(x, t)+B_{y}(x+\Delta, t)\right)\right) \\
= & \frac{1}{\Delta}\left(-\frac{1}{2}\left(E_{z}(x, t)+E_{z}\left(x, t+\Delta_{t}\right)\right)\right. \\
+ & \left.\frac{1}{2}\left(E_{z}(x+\Delta, t)+E_{z}\left(x+\Delta, t+\Delta_{t}\right)\right)\right)
\end{aligned}
$$

For Ampère's law equations similar to (1) and (2) hold. Both the explicit and the implicit method have been successfully coupled with constitutive equations for magnetized plasmas [8], [9], [1], a fact which we will exploit in the numerical examples but not discuss further. Advantages of the fully implicit method include unconditional stability and excellent compatibility with auxiliary differential equations for complex materials [1]. The main disadvantage is, of course, its implicit nature: the need to solve a large (if sparse) set of equations at every time step. This is the main motive for attempting to use it only locally so that we can get the best of both worlds: efficient easily-parallelisable FDTD solutions where possible, combined with implicit highly stable solutions where needed. 

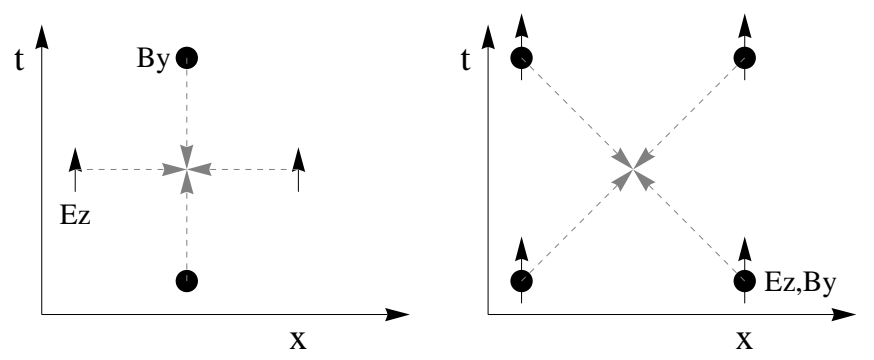

Fig. 1. The staggered FDTD grid (left) has central difference derivatives located where needed. The collocated fully implicit grid (right) enforces equality between a spatially-interpolated temporal central difference and a temporally-interpolated spatial central difference [1].

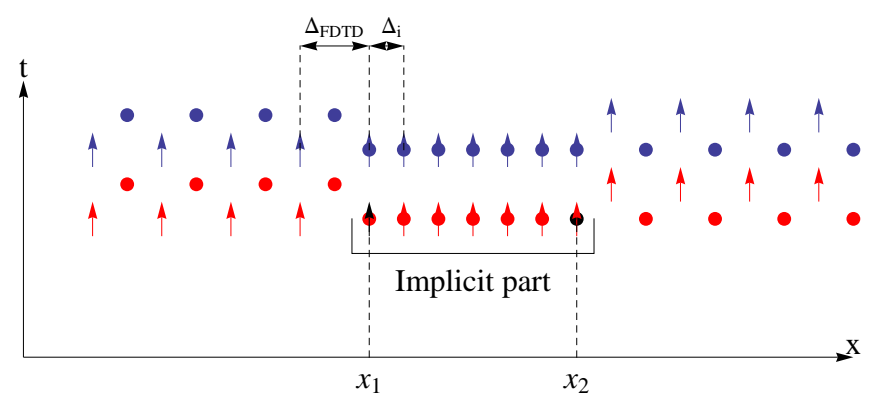

Fig. 2. A fully explicit FDTD scheme can be stably coupled to a fully implicit method. For clarity, $\Delta_{F D T D}=2 \Delta_{i}$ in the figure, but in general $\Delta_{F D T D} \gg \Delta_{i}$

\section{HYBRID ALGORITHM}

In figure 2, an implicit grid with discretisation length $\Delta_{i}$ is combined with two explicit FDTD grids of discretisation length $\Delta_{F D T D}$. There are only two special cases to be considered: one involving the black arrow (electric field) at the left interface $\left(x=x_{1}\right)$, and one involving the black dot (magnetic induction) at the right interface $\left(x=x_{2}\right)$. Both the FDTD method and the fully implicit method can be interpreted as finite-element methods with specific basis- and test-functions [1], [4], a fact which has been used to construct explicit local refinement schemes [4], [10]. Without going into detail, these finite-element interpretations lead to the following discrete equation for the magnetic field at the right interface $\left(x=x_{2}\right)$

$$
\begin{gathered}
\frac{1}{\Delta_{t}}\left(\frac{1}{2}\left(B_{y}\left(x, t+\Delta_{t}\right)+B_{y}\left(x+\Delta_{F D T D}, t+\Delta_{t}\right)\right)\right. \\
\left.-\frac{1}{2}\left(B_{y}(x, t)+B_{y}\left(x+\Delta_{F D T D}, t\right)\right)\right) \\
=\frac{1}{\Delta_{F D T D}}\left(\frac { 1 } { 2 } \left(E_{z}\left(x+\Delta_{F D T D} / 2, t+\Delta_{t} / 2\right)\right.\right. \\
\left.\quad+E_{z}\left(x+3 \Delta_{F D T D} / 2, t+\Delta_{t} / 2\right)\right) \\
\left.\quad-\frac{1}{2}\left(E_{z}(x, t)+E_{z}\left(x, t+\Delta_{t}\right)\right)\right)
\end{gathered}
$$

The left-hand side is the implicit spatial interpolation ([1]) of the time derivative (which also occurs in (2)), this time using 2 implicit and 2 explicit points, which are available at the correct positions. The right-hand side interpolates two explicit
$E_{z}$ values at $\Delta_{F D T D} / 2$ and $3 \Delta_{F D T D} / 2$ from the interface to get $E_{z}$ at $\Delta_{F D T D}$ from the interface. This is used together with two implicit $E_{z}$ values at the interface to end up with a spatial derivative located at the same spatio-temporal position as the interpolated time derivative. A similar equation updates $E_{z}$ at the left interface $\left(x=x_{1}\right)$.

$$
\begin{gathered}
\frac{\epsilon_{0} \mu_{0}}{\Delta_{t}}\left(\frac{1}{2}\left(E_{z}\left(x, t+\Delta_{t}\right)+E_{z}\left(x-\Delta_{F D T D}, t+\Delta_{t}\right)\right)\right. \\
\left.-\frac{1}{2}\left(E_{z}(x, t)+E_{z}\left(x-\Delta_{F D T D}, t\right)\right)\right) \\
=\frac{1}{\Delta_{F D T D}}\left(-\frac{1}{2}\left(B_{y}\left(x-\Delta_{F D T D} / 2, t+\Delta_{t} / 2\right)\right.\right. \\
\left.+B_{y}\left(x-3 \Delta_{F D T D} / 2, t+\Delta_{t} / 2\right)\right) \\
\left.\quad+\frac{1}{2}\left(B_{y}(x, t)+B_{y}\left(x, t+\Delta_{t}\right)\right)\right)
\end{gathered}
$$

With (3) and (4), the algorithm can proceed as follows

1) Explicit update for Faraday's law on the right explicit part, and store one "past" magnetic field point needed for the special case (3).

2) Explicit update for Ampère's law on the left explicit part, and store one "past" electric field point needed for the special case (4).

3) Implicit update in the implicit part, including two special cases.

4) Explicit update for Ampère's law on the right explicit part.

5) Explicit update for Faraday's law on the left explicit part.

This algorithm is stable at the FDTD Courant limit, no matter how small $\Delta_{i}$ is (because of the unconditional stability of the implicit method).

\section{NUMERICAL EXAMPLES}

\section{A. A thin conducting layer}

The implicit region can be used at very small $\Delta_{i}$ to resolve evanescent phenomena with extremely small decay lengths. We used this to calculate the transmission coefficient for a wave traveling through a very thin layer of copper $\left(\sigma=5.96 \cdot 10^{7} \Omega^{-1}\right)$ (figure 3). The thickness of the conductor is $d=10 \mu m=\left(13 \cdot 10^{-5}\right) \Delta_{F D T D}$, far smaller than the FDTD discretisation length. There are 50 implicit points inside this thin conducting layer. The entire algorithm runs at the FDTD Courant limit. The set of equations to be solved is very sparse and banded, and its size only depends on the amount of discretisation points inside the conductor, here $100 \times 100(50$ points, 1 electric and 1 magnetic degree of freedom per point). For well-resolved wavelengths, the analytical and numerical solution are nearly identical. As the frequency increases, the waves become ill-resolved in either the FDTD or the implicit part, and the results become inaccurate.

We also determined the transmission coefficient through a thin conducting layer with parabolic $\sigma$ variation, $(\sigma=$ $\sigma_{\text {copper }}\left(1-(2 x / d)^{2}\right)$ where $d=10 \mu m$ is still the layer thickness). The exact solution can be calculated in terms of parabolic cylinder functions [11], and agrees very well with the 
numerical result. This demonstrates our claim that no detailed knowledge of the physics in the evanescent layer is needed for our method to work.

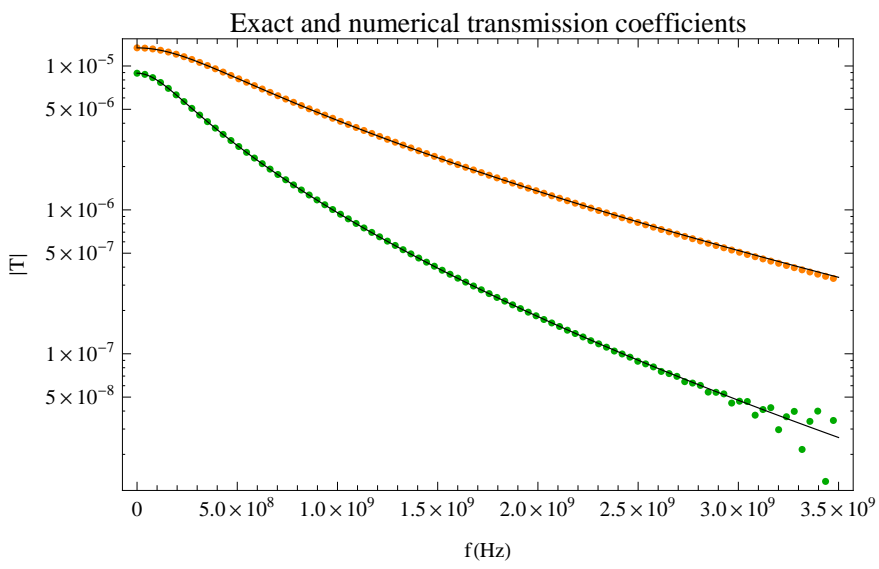

Fig. 3. Exact and numerical transmission coefficient obtained using a Gaussian pulse traveling through a thin layer of copper (green: constant $\sigma$; orange: parabolic $\sigma$ variation).

\section{B. Budden tunneling}

The wave equation

$$
\frac{\partial^{2}}{\partial x^{2}} E+k(x)^{2} E=\frac{\partial^{2}}{\partial x^{2}} E+\left(\frac{\beta}{x}+\frac{\beta^{2}}{\eta^{2}}\right) E=0
$$

can be solved exactly in terms of hypergeometric functions [12]. This is known as Budden tunneling. $k_{\infty}^{2}=\frac{\beta^{2}}{\eta^{2}}$ is the asymptotic (large $|x|$ ) wavenumber. The distance between the zero $k(x)=0$ and the singularity is $\Delta=\frac{\eta^{2}}{\beta}$, from which $\eta=\left|k_{\infty} \Delta\right|$ relates to the amount of asymptotic wavelengths between the zero and the singularity.

Analytical expressions for reflection- and transmissioncoefficients are known. In particular, the absolute value of the transmission coefficient for a left-moving wave is

$$
|T|=\exp \left(-\frac{1}{2} \pi \eta\right)
$$

In plasma physics, Budden tunneling is usually an approximation [13]. It is possible to construct the space-dependence of the density and the background magnetic field in such a way that the cold plasma wave equation becomes the Budden equation (5) exactly. In figure 4 , we have modeled such a plasma $(\beta=1, \eta=1)$. We used our implicit refinement technique near the singularity in the Budden equation (5), and we find that the resulting numerical transmission coefficient is indeed close to the predicted one.

\section{CONClusion}

In this paper, we hybridized the fully explicit FDTD method with the fully implicit method of [1]. We have shown that this can be used to accurately model thin evanescent layers (possibly much shorter than the FDTD discretisation length) leaving the Courant limit unchanged. This method requires no detailed knowledge of the physics in the evanescent region:

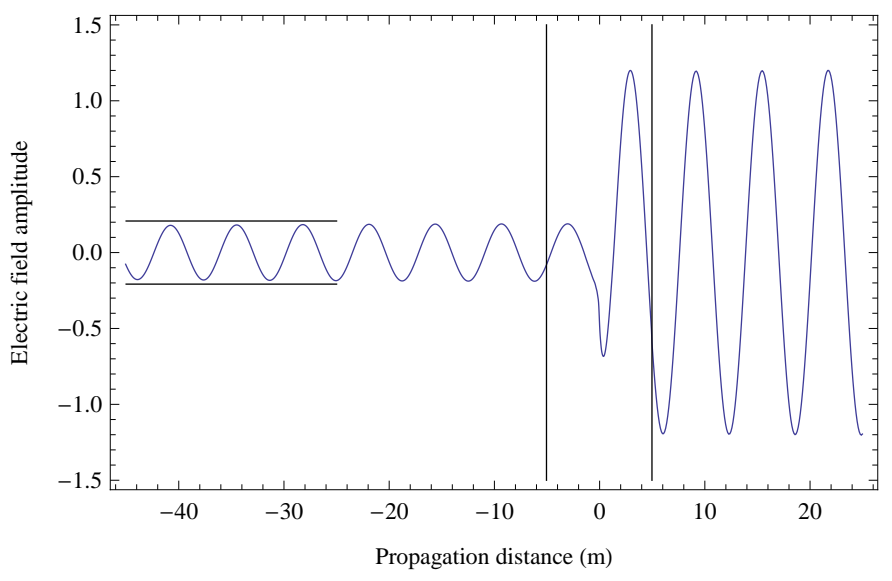

Fig. 4. Budden tunneling. The wave is traveling from right to left. Vertical black lines indicate the refined implicit region near the zero and singularity of the non-uniform wave equation. Horizontal black lines indicate the analytically expected amplitude of the transmitted wave based on Budden theory.

no (approximate) analytical solutions nor Green functions are needed. Further research aims at incorporating this approach in a full 3D FDTD method.

\section{REFERENCES}

[1] W. Tierens and D. D. Zutter, "An unconditionally stable time-domain discretization on cartesian meshes for the simulation of nonuniform magnetized cold plasma," Journal of Computational Physics, pp. 51445156, 2012.

[2] S. V. den Berghe, F. Olyslager, and D. D. Zutter, "Accurate modeling of thin conducting layers in FDTD," IEEE Microwave and Guided Wave Letters, vol. 8, no. 2, pp. 75-77, Feb. 1998.

[3] S. V. den Berghe, "Object-oriented electromagnetic simulations with the finite-difference time-domain method," Ph.D. dissertation, Ghent University, 2000.

[4] R. A. Chilton, "H-, P- and T-refinement strategies for the finitedifference-time-domain (FDTD) method developed via finite-element (FE) principles," Ph.D. dissertation, Ohio State University, 2008.

[5] F. Collino, T. Fouquet, and P. Joly, "Conservative space-time mesh refinement methods for the FDTD solution of Maxwell's equations," Journal of Computational Physics, pp. 9-35, 2005.

[6] S. Piperno, "Symplectic local time-stepping in non-dissipative DGTD methods applied to wave propagation problems." RAIRO Model Math. Anal. Numer, pp. 3-30, 2005.

[7] A. Taflove and S. Hagness, Computational Electrodynamics: The FiniteDifference Time-Domain Method. Artech House, 2005.

[8] D. N. Smithe, "Finite-difference time-domain simulation of fusion plasmas at radiofrequency time scales," Physics of Plasmas, vol. 14, no. 14, pp. 2537-2549, Apr. 2007.

[9] J. Young, "A full finite difference time domain implementation for radio wave propagation in a plasma," Radio science, pp. 1513-1522, 1994.

[10] W. Tierens and D. D. Zutter, "BOR-FDTD subgridding based on finite element principles," Journal of Computational Physics, pp. 4519-4535, 2011.

[11] M. Abramowitz and I. Stegun, Handbook of Mathematical Functions with Formulas, Graphs, and Mathematical Tables. Dover Publications, 1964.

[12] T. Stix, Waves in Plasmas. American Institute of Physics, 1992.

[13] Y. Kazakov, I. Pavlenko, and I. Girka, "Propagation of the fast magnetosonic wave through the generalized budden barrier," ВОПРОСЫ АТОМНОЙ НАУКИ И ТЕХНИКИ, pp. 90-93, 2010. 